\title{
A METRIZATION THEOREM
}

JANET S. ALLSBROOK

A characterization of metrizable topological spaces in terms of subtopologies is given. First, several terms are defined in order to describe the pertinent subtopologies. Then, the characterization is readily established as a result of a metrization theorem due to Bing [1] and a metrization theorem due to Ceder [2].

Definition 1. Let $(X, \mathfrak{J})$ be a topological space having the property that the intersection of an arbitrary number of open sets is open. Then $(X, J)$ is called a fundamental topological space.

Definition 2. Let $(X, J)$ be a topological space with a base $\beta=\left\{B_{j}: j \in J\right\}$ such that for each $x \in \cap\left\{B_{i}: i \in I, I\right.$ an arbitrary subset of $J\}$ there exists $B_{x} \in \beta$ where $x \in B_{x} \subset \cap\left\{B_{i}: i \in I\right\}$. Then, $\beta$ is called a fundamental base for $J$.

REMARK. If $(X, \mathfrak{J})$ has a fundamental base for $\mathfrak{J}$, it is a fundamental space.

Definition 3. Let $(X, \Im)$ be a topological space. Let $\beta_{i}$, a subcollection of $J$, be a base for some topology, $\Im_{i}$, on $X$. Consider the subcollection of $J, \bar{\beta}_{i}$, where $\bar{\beta}_{i}=\left\{X-\bar{T}_{i}: T_{i} \in J_{i}\right.$ and the closure is with respect to $J\}$. Then, $\bar{\beta}_{i}$ is a base for a topology on $X, J / J_{i}$. The topology, $J / J_{i}$ is called the dual topology of $\mathcal{J}_{i}$ relative to $J$. $\bar{\beta}_{i}$ is called the dual base of $\beta_{i}$.

We state two metrization theorems, without proof, to be used in the establishment of a new metrization theorem. For other proofs see $[1],[2]$.

A Metrization Theorem of Bing. A topological space is metrizable if and only if it is $T_{1}$ and regular and the topology has a $\sigma$-discrete base.

A Metrization Theorem of Ceder. A topological space $(X, \mathrm{~J})$ is metrizable if and only if it is $T_{1}$ and regular and the topology has a $\sigma$-closure preserving base, $\beta=\cup_{i=1}^{\infty}\left\{B_{i}\right\}$, such that for each $x \in X$, and $n, a$ positive integer, $\cap\left\{B: x \in B, B \in B_{n}\right\}$ is a neighborhood of $x$.

We now state a metrization theorem which characterizes a topological space, $(X, \Im)$, as metrizable in terms of a countable family of bases, $\beta_{i}, i=1,2, \cdots$ for subtopologies, $\Im_{i}$ of $\mathfrak{J}$, and in terms of the dual bases, $\bar{\beta}_{i}, i=1,2, \cdots$. More precisely:

Theorem. A topological space $(X, J)$ is metrizable if and only if it is $T_{1}$ and regular and has a base $\beta=\bigcup_{i=1}^{\infty} \beta_{i}$ where for each $i, \beta_{i}$ is a fun-

Received by the editors July 29, 1964. 
damental base for a topology, $\Im_{i}$, on $X$, and for each $i$, the dual base, $\bar{\beta}_{i}$ is a fundamental base for $J / J_{j}$, the dual topology of $\Im_{i}$ relative to $J$.

Proof. Let $(X, J)$ be a topological space satisfying the hypotheses above. Then, we shall show that $(X, J)$ satisfied the hypotheses of Ceder's Theorem. Let $\beta=\bigcup_{i=1}^{\infty} \beta_{i}$ be the given base for J. Then, for each $x \in X, n$ a positive integer, $\cap\left\{B: x \in B, B \in \beta_{n}\right\}$ is a neighborhood of $x$ in $\Im_{n} \subset$ J. Also, $X-\cup\left\{\bar{B}_{i}: B_{i} \in \beta_{n}^{\prime} \subset \beta_{n}\right\}=\bigcap\left\{X-\bar{B}_{i}\right\}$ $\in J / J_{i} \subset J$. Therefore, $U\left\{\bar{B}_{i}\right\}$ is closed. Hence, $\cup\left\{\bar{B}_{i}\right\}=\operatorname{cl}\left[\cup B_{i}\right]$. Thus, $\beta_{n}$ is a closure preserving family.

Now, let $(X, J)$ satisfy the hypotheses of Bing's Theorem, and let $\beta=\bigcup_{i=1}^{\infty} \beta_{i}$ be the $\sigma$-discrete base. We define $\beta_{i}^{\prime}=\beta_{i} \cup\{X\}, i=1,2, \cdots$ It is apparent that $\beta_{i}^{\prime}$ is a fundamental base for some subtopology, $J_{i}^{\prime}$, of $\zeta$ for each $i$. It remains only to show that $\bar{\beta}_{i}^{\prime}$ is a fundamental base, also. Now, $\cap_{\alpha}\left\{X-\bar{T}_{\alpha}: T_{\alpha} \in J_{i}^{\prime}\right\}=X-\bigcup_{\alpha} \bar{T}_{\alpha}=X-\operatorname{cl}\left[\cup_{\alpha} T_{\alpha}\right]$ $\in J / J_{i}^{\prime}$, since $\beta_{i}^{\prime}$ is a closure preserving subfamily of $\mathfrak{J}$ implies that $\Im_{i}^{\prime}$ is also a closure preserving subfamily of $\Im$. Thus, $\bar{\beta}_{i}^{\prime}$ is a fundamental base.

\section{REFERENCES}

1. R. H. Bing, Metrization of topological spaces, Canad. J. Math. 3 (1951), 175-186.

2. J. G. Ceder, Some generalizations of metric spaces, Pacific J. Math. 11(1961), $105-125$.

UNIVERSITY OF FLORIDA 